\title{
Review Article \\ SIRT1 Regulates Cognitive Performance and Ability of Learning and Memory in Diabetic and Nondiabetic Models
}

\author{
Yue Cao, Zi Yan, Tong Zhou, and Guixia Wang \\ Department of Endocrinology and Metabolism, The First Hospital of Jilin University, Changchun, Jilin, China \\ Correspondence should be addressed to Guixia Wang; gwang168@jlu.edu.cn
}

Received 20 June 2017; Accepted 8 August 2017; Published 15 October 2017

Academic Editor: Judy de Haan

Copyright @ 2017 Yue Cao et al. This is an open access article distributed under the Creative Commons Attribution License, which permits unrestricted use, distribution, and reproduction in any medium, provided the original work is properly cited.

\begin{abstract}
Type 2 diabetes mellitus is a complex age-related metabolic disease. Cognitive dysfunction and learning and memory deficits are main characteristics of age-related metabolic diseases in the central nervous system. The underlying mechanisms contributing to cognitive decline are complex, especially cognitive dysfunction associated with type 2 diabetes mellitus. SIRT1, as one of the modulators in insulin resistance, is indispensable for learning and memory. In the present study, deacetylation, oxidative stress, mitochondrial dysfunction, inflammation, microRNA, and tau phosphorylation are considered in the context of mechanism and significance of SIRT1 in learning and memory in diabetic and nondiabetic murine models. In addition, future research directions in this field are discussed, including therapeutic potential of its activator, resveratrol, and application of other compounds in cognitive improvement. Our findings suggest that SIRT1 might be a potential therapeutic target for the treatment of cognitive impairment induced by type 2 diabetes mellitus.
\end{abstract}

\section{Introduction}

Type 2 diabetes mellitus (T2DM) is one of multiple agerelated metabolic diseases [1]. Several latest studies have demonstrated severe and progressive abnormalities in brain structures and cognition during the early stage of T2DM [2]. T2DM is a risk factor for mild cognitive impairment (MCI) [3] and can accelerate the rate of functional decline in patients with mild dementia [4]. Cognitive dysfunction and learning and memory deficits have been considered one of the most prevalent and significant T2DM-related complications [4-8]. In recent years, silent information regulator 2 (Sir2), the highly conserved nicotinamide adenine dinucleotide- $\left(\mathrm{NAD}^{+}-\right)$dependent histone deacetylase [9], was shown to extend lifespan and delay aging in numerous studies ranging from Saccharomyces cerevisiae to mammals $[10,11]$. As the ortholog of the yeast Sir2, SIRT1 is the most evolutionally conserved member [12]. Accumulating evidence has suggested that SIRT1 is expressed in the liver, skeletal muscle, pancreas, adipose tissues, and brain $[13,14]$, but its levels in the brain are notably higher than those in the other tissues in mammals $[12,15,16]$, especially in the hippocampus, a vital structure closely related to learning and memory of the central nervous system [17]. SIRT1 participates in apoptosis [18], autophagy [19], and development [20], as well as in metabolism [21,22] and circadian rhythms $[23,24]$; therefore, it is not surprising that SIRT1 affects more complex biological processes including aging [24-27], MCI [28], and cognitive decline [29-31].

Present opinion on SIRT1 in cognition, learning, and memory is inconclusive. Some scholars believe that SIRT1 is positive for memory conservation. The spontaneous senescence-accelerated P8 mouse strain (SAMP8) is widely used as an animal model of aging [32-34] due to learning and memory deficits and behavioral alterations of Alzheimer's disease $(\mathrm{AD})$ [35-39]. It has been demonstrated that the expression of SIRT1 declines with age in the brain of SAMP8 and senescence-accelerated mouse resistant 1 (SAMR1) [40], which have been extensively used as a control model because of the same genetic background and normal aging characteristics [41]. However, SIRT1 was decreased in the cerebral cortex and hippocampus [42] of SAMP8 mice [43,44] compared with those of age-matched SAMR1. In addition, SIRT1 was downregulated in diverse models of cognitive impairment 
in vivo and in vitro, such as in juvenile C57BL/6J mice with dysmetabolism induced by high-caloric diet [45] and neurotoxic primary hippocampal neurons caused by toxins [44]. A study by Yokozawa et al. on antiaging effects of oligomeric proanthocyanidins found that SIRT1 was increased both in the cellular senescence model [46] and SAMP8 mouse model [47]. On the other hand, some researchers believed that SIRT1 has no effect on cognitive improvement and has a counterproductive effect. Most tellingly, earlier studies have proved that overexpression of SIRT1 may induce the memory deficit in transgenic ( $\mathrm{Tg}$ ) mice that overexpresses human SIRT1 in neurons [48]. Nicotinamide, an inhibitor of SIRT1, has been shown to attenuate cognitive deficits of $3 \times \mathrm{Tg}-\mathrm{AD}$ mice via inhibition of SIRT1 and phosphorylation of tau [49]. Moreover, recent work has demonstrated that SIRT1 silencing could promote neuronal survival and protect neurons via the IGF-1 pathway [50].

Collectively, SIRT1 plays a significant role in learning and memory and provides enormous insights into T2DMassociated cognitive dysfunction. It is also rapidly emerging as a critical regulator of aging. However, positive or negative effects of SIRT1 on learning and memory have yet to be further discussed. In the ensuing paragraphs, we highlighted the involvement of SIRT1 in pathological processes of cognitive impairment in diabetic and nondiabetic models.

\section{Role of SIRT1 on Cognition and Learning and Memory in Nondiabetic Models}

2.1. Deacetylation of SIRT1. Many studies have confirmed that SIRT1 mediates chromatin silencing and chromatin remodeling through deacetylating histones, including $\mathrm{H} 1$, $\mathrm{H} 3$, and $\mathrm{H} 4$ [51] and modulates the activity of several protein targets that will be stated subsequently.

2.1.1. SIRT1-p53 Pathway. It has been claimed that SIRT1 directly bound to and deacetylated p53 with specificity for its C-terminal Lys382 residue, inhibited acetylation of p53, and reduced the activity of downstream target genes [52, 53].

Decreased level of SIRT1 and increased level of acetylated p53 were observed in the hippocampal tissue [54] and cortex [55] of SAMP8 and in vitro studies [56]. Coincidentally, in juvenile C57BL/6J mice, low-caloric intake increased learning and memory function through positively downregulating p53 and unremarkably upregulating SIRT1 [45]. In the following studies, although no difference in SIRT1 level was detected between the control and the resveratrol dietary groups, researchers found that resveratrol improved learning and memory through the SIRT1-p53 pathway [57].

2.1.2. SIRT1-AMPK Pathway. SIRT1 improves mitochondrial function by activating adenosine monophosphateactivated protein kinase (AMPK) through acetylating liver kinase B1 (LKB1) [58]. Conversely, AMPK improves SIRT1 activity by increasing cellular $\mathrm{NAD}^{+}$levels to trigger the deacetylation of SIRT1 [59]. In the SAMP8 model, the increases in phosphorylated AMPK (p-AMPK) that regulate energy expenditure and the decreases in the production of reactive oxygen species (ROS) paralleled to the rise in SIRT1 in the hippocampus [54] and cortex [55]. Although without detection of LKB1, we suggest that this process might be triggered by SIRT1 deacetylation. In addition, in a rat model of $\mathrm{AD}$ with intracerebroventricular injection of streptozotocin (ICV-STZ) [60], the level of p-AMPK and SIRT1 activity were decreased and the level of phosphorylated tau was increased, while AMPK-specific activator prevented cognitive impairment through rescuing SIRT1 activity, downregulating tau hyperphosphorylation, and repairing mitochondrial function reflected by increased ATP levels, mitochondrial membrane potential, complex I activity, and SOD activity, as well as decreased ROS generation.

2.1.3. Other Factors Deacetylated by SIRT1. In addition to the tumor suppressor factor p53 [61, 62] and serine-threonine protein kinase LKB1 [58, 63], SIRT1 deacetylated several transcriptional factors participated in transcriptional control of key genes in multiple cellular processes. These transcriptional factors regulate a wide range of metabolic activities, such as nuclear factor-kappa beta $(\mathrm{NF} \kappa \beta)$ [64], extracellular signalregulated kinase (ERK) [65], the forkhead box subgroup O (FoxO) family $[66,67]$, peroxisome proliferator-activated receptors $\gamma(\operatorname{PPAR} \gamma)$, and its transcriptional coactivator PPAR $\gamma$ coactivator $1-\alpha$ (PGC- $1 \alpha)[68,69]$.

Direct in vivo evidence supported the link between SIRT1 and improvement of cognitive decline. The spatial memory deficit of ICV-STZ-treated rats was improved through ameliorating activation of SIRT1, which in turn attenuated tau phosphorylation by decreasing ERK1/2 phosphorylation [65]. In a study on neuroprotective role of intermittent fasting (IF) [70], upregulation of SIRT1 in the cortex and hippocampus of SAMP8 could possess neuroprotection via modulating downstream factors, including a decrease in phosphorylated Jun-terminal kinase (JNK), acetylated NFK $\beta$ [71], and acetylated FoxO1, as well as an increase in phosphorylated FoxO1. Additionally, in the hippocampus and cortex of SAMP8 mice [71] and in the hippocampus of $3 \times$ Tg-AD mice [72], SIRT1 upregulated a disintegrin and metalloprotease 10 (ADAM10) [73] and downregulated the phosphorylated form of glycogen synthesis kinase 3 beta $(\mathrm{GSK} 3 \beta)[55,70]$ in order to reduce the production of amyloid beta $(\mathrm{A} \beta)$ peptides and tau phosphorylation, which have been widely accepted as vital causes of cognitive decline [74]. SIRT1 was also noted to increase the expression of heat shock protein 70 (HSP70), a biomarker of neuronal survival, in SAMP8 models [70] and 3xTg-AD mice [72].

Moreover, an indirect proof of the effect of SIRT1 on cognition was demonstrated in vitro. In a study on neurite outgrowth and cell survival, SIRT1 was shown to promote neuronal growth through negative modulation of the mammalian target of rapamycin (mTOR)/p70S6 kinase (p70S6K) pathway in wild-type mouse primary neurons and human SIRT1 transgenic mice [75]. Furthermore, Codocedo et al. have suggested that SIRT1 accelerated the development and maintenance of dendritic branching in Sprague-Dawley rat primary hippocampal neurons by inhibiting the RhoA/Rho-associated protein kinase (ROCK) pathway and activating the Racl/JNK pathway [76]. 


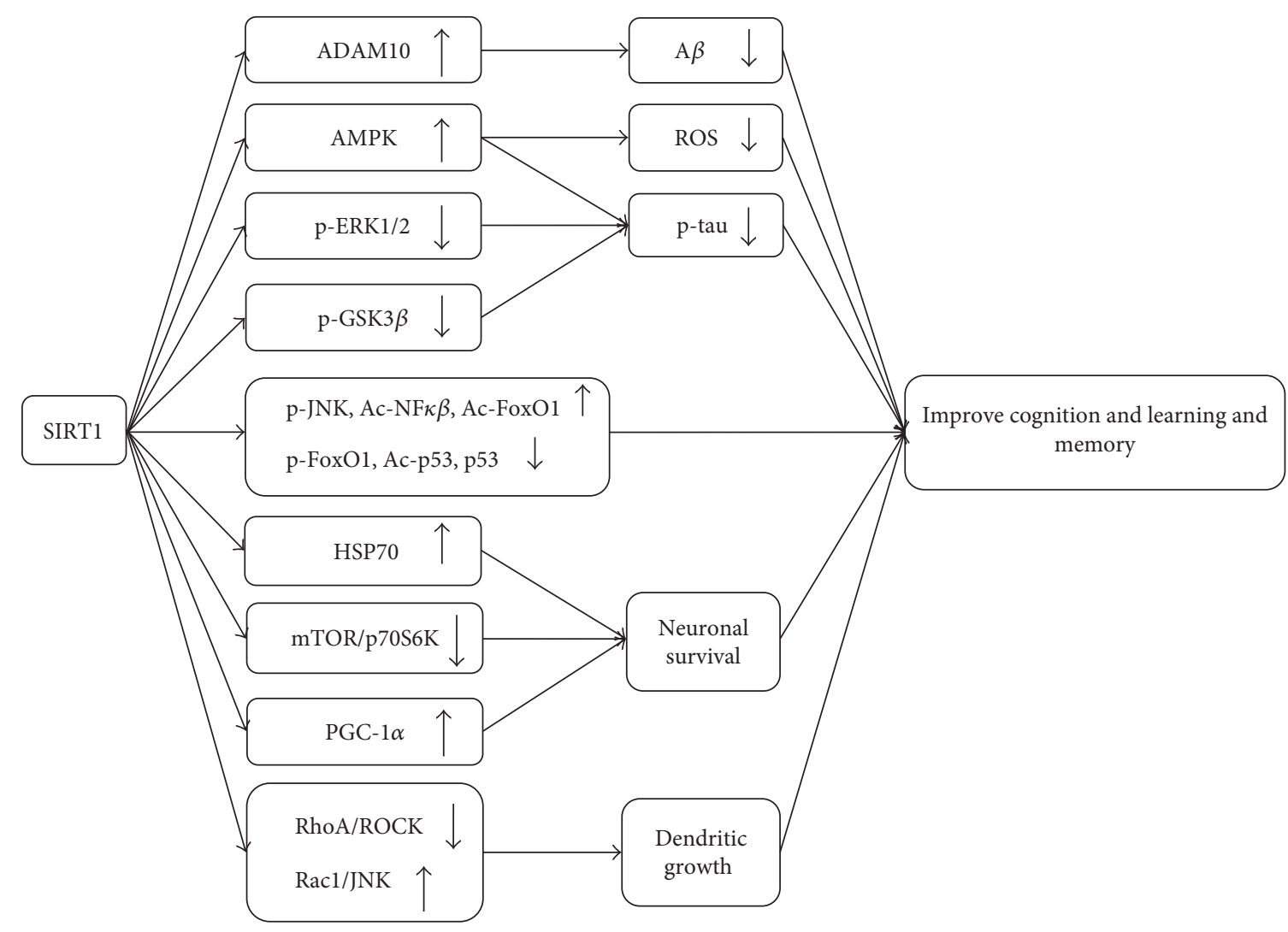

FIGURE 1: Deacetylation of SIRT1 in cognition and learning and memory. Increased SIRT1 level may reduce the production of ROS, A $\beta$, and p-tau, as well as promote neuronal survival and dendritic growth, contributing to improve learning and memory. ADAM10: a disintegrin and metalloprotease 10; AMPK: adenosine monophosphate-activated protein kinase; p-ERK: phosphorylated extracellular signal-regulated kinase; p-GSK3 $\beta$ : phosphorylated glycogen synthesis kinase 3 beta; p-JNK: phosphorylated Jun-terminal kinase; Ac-NF $\kappa \beta$ : acetylated nuclear factor-kappa beta; Ac-FoxO1: acetylated the forkhead box subgroup O 1; Ac-p53: acetylated p53; HSP70: heat shock protein 70; mTOR: mammalian target of rapamycin; p70S6K: p70S6 kinase; PGC- $1 \alpha$ : peroxisome proliferator-activated receptor $\gamma$ transcriptional coactivator 1- $\alpha$; ROCK: Rho-associated protein kinase; JNK: Jun N-terminal kinase; $A \beta$ : amyloid beta; ROS: reactive oxygen species; p-tau: phosphorylated tau.

SIRT1was shown to attenuate glutamate-induced apoptosis in SH-SY5Y cells by upregulating PGC-1 $\alpha$ [77].

Such a point is worthy of further confirmation since growing evidence has indicated the presence of relationship between the role of SIRT1 on learning and memory and histone $\mathrm{H} 2 \mathrm{~A}$ variant, H2A.Z, which has been considered a negative regulator of consolidation of recent and remote memory [78]. H2A.Z was negatively regulated by the expression and activity of SIRT1 in some tissues [79] (Figure 1).

2.2. Targeting Oxidative Stress (OS). As the basis of aging theories [80], OS can trigger the pathological processes of learning and memory deficits $[81,82]$. A series of biomarkers represent the degree of OS, such as superoxide dismutase (SOD), reactive oxygen species (ROS), and malondialdehyde (MDA). Mitochondrial dysfunction is the central to oxidative damage and reflects the aging processes [83].

2.2.1. Amelioration of Mitochondrial Dysfunction by SIRT1. There is a growing body of evidence supporting that mitochondrial dysfunction is critical for synaptic aging induced by chronic OS [82]. Data gathered from diverse studies have confirmed that oxidative stress could cause damages in the brain of SAMP8 mice $[84,85]$. SAMP8 primary neurons had poor mitochondrial function, lower mitochondrial membrane potential, and higher mitochondrial vulnerability, all of which was protected by increased SIRT1 expression [56]. In addition, electron transport chain (ETC) related to mitochondrial oxidative phosphorylation (OXPHOS) was changed in vitro [56]. It was remarkable to find that SIRT1 could enhance OXPHOS via increasing the electronic chain-specific components ranging from complex I to complex V in the hippocampus of SAMP8 [54]. Consistent with this notion, SIRT1 improved spatial learning and memory deficits via SIRT1-mediated antioxidant signaling pathways in the D-galactose-induced aging rats [86]. Manganese superoxide dismutase (Mn-SOD) is an important antioxidative enzyme present in mitochondria. Recent data have shown downregulation of Mn-SOD mRNA levels by increasing level of SIRT1 [86].

2.2.2. Interaction of ROS, Inflammatory Factor, and SIRT1. Lower levels of SOD, as well as higher levels of ROS [87], MDA [88], and some proinflammatory factors [ 87,88$]$, were 


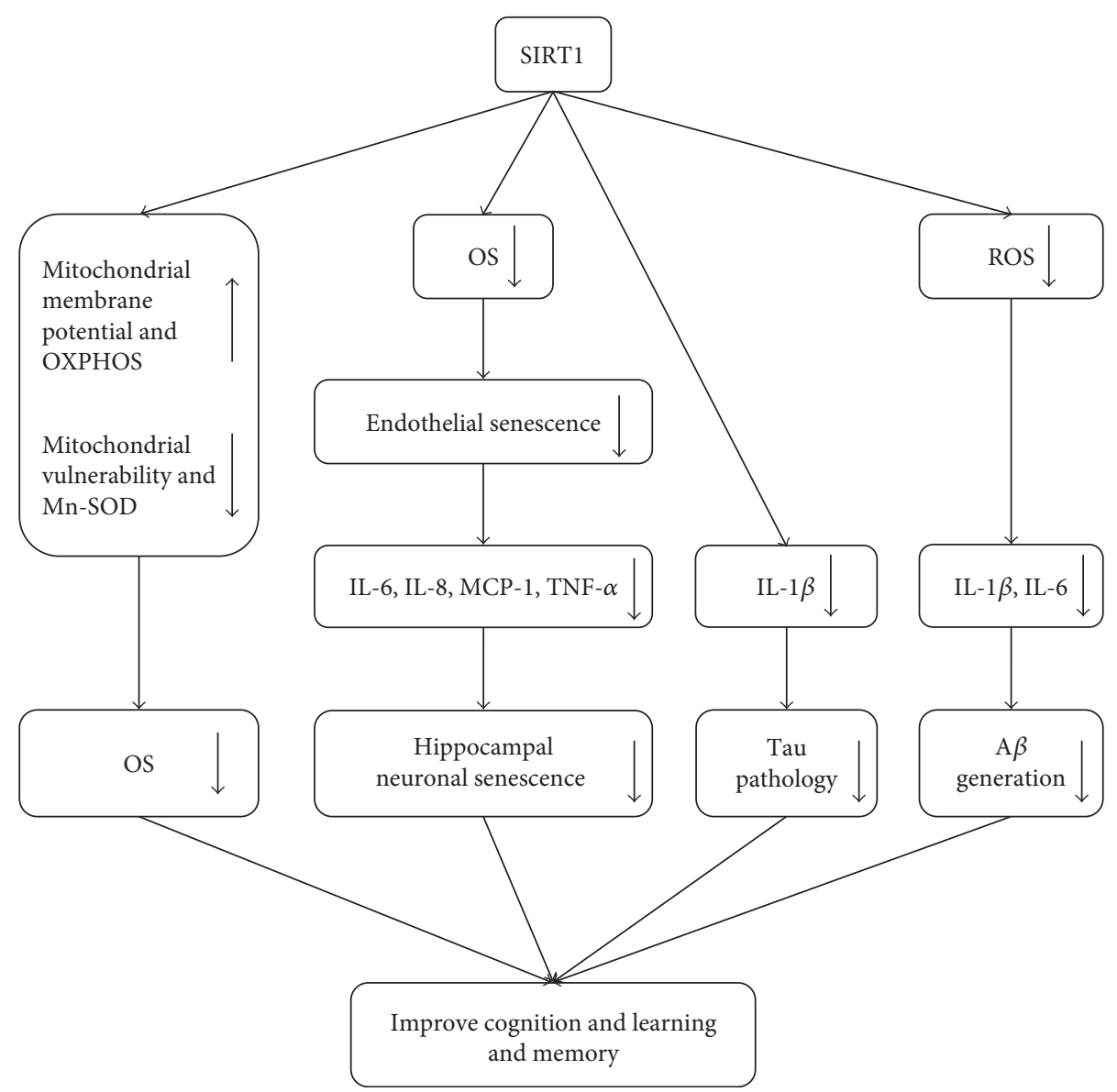

FIGURE 2: Oxidative stress (OS) is regulated by SIRT1 in cognition and learning and memory. SIRT1 may improve learning and memory by inhibiting OS, inhibiting the inflammatory response and hippocampal neuronal senescence, and decreasing the expression of tau and A $\beta$. OS: oxidative stress; OXPHOS: oxidative phosphorylation; Mn-SOD: manganese superoxide dismutase; IL-6: interleukin-6; IL-8: interleukin-8; MCP-1: monocyte chemoattractant protein-1; TNF- $\alpha$ : tumor necrosis factor- $\alpha$; IL- $1 \beta$ : interleukin- $1 \beta$; ROS: reactive oxygen species; A $\beta$ : amyloid beta.

found in SAMP8 compared with age-matched SAMR1. OS aggravated cognitive loss in SAMP8 models through either generating $\mathrm{A} \beta_{1-40}$ and $\mathrm{A} \beta_{1-42}$ by releasing interleukin- $1 \beta$ (IL-1 $\beta$ ) and interleukin-6 (IL-6) [87] or enhancing neuroinflammatory activity by increasing IL- $1 \beta$, tumor necrosis factor- $\alpha$ (TNF- $\alpha$ ), and IL-6 [88]. In line with above evidence, in vitro, senescent endothelial cells induced by OS promoted the senescence of hippocampus neuronal cells through secretion of several inflammatory cytokines such as IL-6, interleukin-8 (IL-8), monocyte chemoattractant protein1(MCP-1), and TNF- $\alpha$ [85]. Upregulation of SIRT1 could reverse inflammatory factors to rescue the production of $\mathrm{A} \beta$ and neuronal senescence [85, 87]. In addition, microglial SIRT1 deficiency elevated levels of IL- $1 \beta$ and exacerbated memory deficits in human P301S tau mice [29] exhibiting age-dependent synaptic loss and tau-mediated memory deficits [89]. All above have demonstrated that neuroinflammatory played a significant role in learning and memory modulated by SIRT1 (Figure 2).

2.3. SIRT1-microRNA Pathway. It is assumed that cyclic AMP response element-binding protein (CREB), a molecular switch of long-term memory that maintains cognitive function [90], binds to several promoters of brain-derived neurotrophic factor (BDNF) and regulates its expression. Recent studies have shown that SIRT1 promotes plasticity and memory in a direct manner via a miR-134-mediated posttranscriptional mechanism. The results suggested that SIRT1 cooperated with Yin Yang 1 (YY1) in binding to the upstream regulatory elements of miR-134 and then limited the expression of miR-134 resulting in overexpression of CREB and BDNF, thereby regulating synaptic plasticity and long-term memory formation in SIRT1-KO mice [91]. Additionally, resveratrol was shown to improve learning and memory in normally aged C57BL/6J mice through the SIRT1-microRNA pathway [92]. Furthermore, SIRT1 increased the expression of BDNF in SAMP8 models [70] and $3 \times T g-A D$ mice [72]. In the hippocampus of rats receiving lead exposure, SIRT1 and CREB phosphorylation were decreased in a dose-dependent manner, which could be reversed by resveratrol [93]. Resveratrol also ameliorated spatial learning memory impairment induced by $A \beta_{1-42}$ in rat hippocampus by elevating SIRT1 expression and CREB phosphorylation [31]. Although miR-134 was not detected 


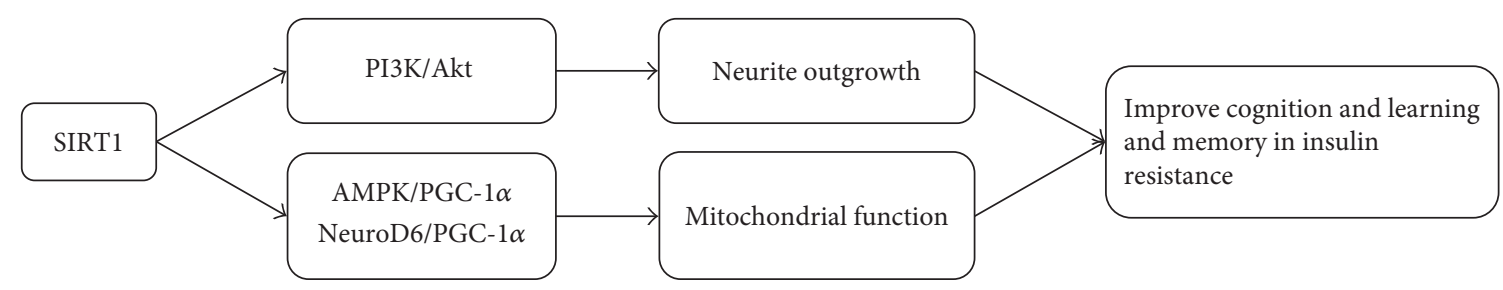

FIGURE 3: The role of SIRT1 in cognition and learning and memory under the condition of IR. SIRT1 has been shown to increase neurite outgrowth by activating the PI3K/Akt pathway and improve mitochondrial function through the AMPK/PGC-1 $\alpha$ and NeuroD6/PGC-1 $\alpha$ pathways. PI3K: phosphoinositide 3-kinase; AMPK: adenosine monophosphate-activated protein kinase; PGC-1 $\alpha$ : PPAR $\gamma$ coactivator 1- $\alpha$; NeuroD6: neuronal differentiation 6.

in the above three studies, we still suggested that SIRT1 protects learning and memory via the SIRT1-miR-134 pathway.

\section{The Mechanism of SIRT1 on Cognition and Learning and Memory under the Condition of Insulin Resistance (IR)}

In the above section, we have summarized the role of SIRT1 in cognitive dysfunction and learning and memory deficits under normal physiological condition. Next, we will discuss its role under the condition of insulin resistance. It is widely known that caloric restriction (CR) has benefits on cognition decline [94]. Emerging evidence has indicated a causal link between T2DM and cognition decline and learning and memory deficits [3-5, 95-97], such as MCI [98]. The mechanisms that trigger learning and memory deficits in diabetic models include inflammation [99], loss of neuronal plasticity $[100,101]$, alteration of mitochondrial structure and function $[102,103]$, elevation of cerebral $\mathrm{A} \beta$, and tau phosphorylation [100]. Therefore, cognitive ability is distinctly affected by metabolic status.

Accumulating evidence has indicated the inhibition of SIRT1 protein expression and activity in T2DM or IR [104-107]. Data has shown that activated SIRT1 improves the insulin sensitivity of the liver, skeletal muscle, and adipose tissues, as well as protects the function and cell mass of pancreatic $\beta$-cells [13]. So, does SIRT1 involve in it? And whether SIRT1 regulates learning and memory directly or indirectly? Next, we set forth the role of SIRT1 in cognition and learning and memory under the condition of IR (Figure 3).

3.1. SIRT1 Promotes Neurite Outgrowth. Several studies have demonstrated that SIRT1 modulates neuronal viability $[36,37]$, neuronal differentiation [38-41], neuronal protection [42-44], and synaptic plasticity [21, 45-47], all of which are key factors largely linked to cognitive improvement. It is well established that insulin exerts its actions in a series of biological processes through binding to insulin receptors [108], as well as plays an essential role in IR and T2DM. Recently, researchers have demonstrated that insulin-induced neurite outgrowth is regulated by SIRT1, which is dependent on the PI3K/Akt signaling pathway in SH-SY5Y cells [109]. In accordance with the above views, we suggest that SIRT1 may be imbalanced when insulin signaling is impaired and cause an influence on cognition and neurodegeneration.
3.2. SIRT1 Improves Mitochondrial Function in the Brain. SIRT1 activation has a significant coordinating role in mitochondrial function. It is noteworthy that NeuroD6, as a regulator of ROS homeostasis [110], is related to learning and memory. As a marker of mitochondrial biogenesis, PGC- $1 \alpha$ may take part in cognitive decline under metabolic stress. Moreover, AMPK is a sensor key that controls PGC$1 \alpha$ activity. In the aged C57BL/6J mouse model of IR induced by a high-fat diet, SIRT1 improved mitochondrial function via the SIRT1-AMPK-PGC- $1 \alpha$ axis and the neuronal differentiation 6 (NeuroD6)-PGC- $1 \alpha$-SIRT1 axis to enhance cognitive decline [102]. Coincidentally, the SIRT1-AMPKPGC- $1 \alpha$ pathway was also verified in the SAMP8 model of IR induced by a high-fat diet, albeit the levels of SIRT1 were not significantly modified [103].

A study by Lennox et al. has demonstrated that increased SIRT1 enhanced cognitive function and synaptic plasticity via alleviating IR in high-fat-fed mice [111]. Similarly, upregulated SIRT1 simultaneously improved synaptic plasticity and insulin signaling in the hippocampus and cortex of high-fat-fed mice [112]. Although few studies have examined the association between IR and cognitive impairment, we concluded that SIRT1 might contribute indirectly to improve cognition, because many of SIRT1's downstream regulators are involved in memory processes.

\section{The Effects of Resveratrol on Cognition and Learning and Memory}

4.1. Resveratrol, Targeting SIRT1 or Not? Resveratrol has attracted considerable attention for its effects on the improvement of IR [113], cognitive decline [31, 65, 114-116], and cardiovascular diseases $[117,118]$. Although resveratrol is widely accepted as a natural activator of SIRT1, there is also evidence showing that resveratrol may not be the direct agonist of SIRT1.

In vitro, resveratrol regulates brain function through increasing the biogenesis of $\alpha$-amino-3-hydroxy-5-methyl4-isoxazolepropionic acid receptor (AMPAR), a glutamatergic receptor, mediated by AMPK and subsequent downstream $\mathrm{PI} 3 \mathrm{~K} / \mathrm{Akt}$ signaling in rat primary neurons [119]. In vivo, resveratrol improves learning and memory through activating the IGF-1-PI3K-p-CREB signaling pathway in the hippocampal CA1 region of juvenile and healthy C57BL/6J mice [57], while maintaining the same expression level of SIRT1. In the same strain with isoflurane-induced cognitive impairment, 
Li et al. have found that resveratrol exerts anti-inflammatory and antiapoptotic actions to recover cognition without alteration of SIRT1 [120]. In their work, the factors related to neuroapoptosis were changed, such as downregulation of cleaved caspase-3 and Bax and upregulation of Bcl-2. Meanwhile, NLRP3, an intracellular receptor of inflammatory responses, IL- $1 \beta$, and TNF- $\alpha$ were decreased.

The effects of resveratrol on cognitive improvement are likely not to be fully dependent on SIRT1. After treatment with resveratrol, activation of the $\mathrm{Wnt} / \beta$-catenin pathway by increasing GSK- $3 \beta$ might as well protect cognitive disturbances in diabetic C57BL/6J mice [102] and SAMP8 mice under the condition of metabolic stress induced by a high-fat diet [103]. In the latter study, Palomera-Avalos et al. have put forward that resveratrol improves mitochondrial morphology, dynamics, and OXPHOS via a decrease in mitofusin 2 (MFN2) and an increase in optic atrophy-1 protein (OPA1), I-NDUFB8, II-SDNB, III-UQCRC2, VATPase complexes, and voltage-dependent anion channel 1 (VDAC1)/porin [103].

4.2. Resveratrol, Improving Cognition and Learning and Memory or Not? According to above notions, resveratrol plays a significant role in cognitive enhancement. However, its effects on cognition and learning and memory are still controversial.

4.2.1. The Effects of Resveratrol on Animal Models. In several studies, the administration of oligomeric proanthocyanidins [47], pterostilbene [121], and rapamycin [122] displayed antiaging effects, whereas resveratrol did not show a marked effect. In the aspect of improvement of spatial learning and memory, five-week resveratrol administration to SAMP8 mice ( $90 \mu \mathrm{mol} / \mathrm{kg}$ body weight/day, about equal to $20 \mathrm{mg} / \mathrm{kg}$ body weight/day) showed no significant changes compared to oligomer administration with the same period $(50 \mathrm{mg} / \mathrm{kg}$ body weight/day). Similarly, eight-week pterostilbene administration to SAMP8 mice $(120 \mathrm{mg} / \mathrm{kg}$ body weight/day) exerted beneficial effects on learning and memory, but not resveratrol at an identical dose for 8 weeks. In the aspect of survival, resveratrol administration $(50 \mathrm{mg} / \mathrm{kg}$ body weight/day and $200 \mathrm{mg} / \mathrm{kg}$ body weight/day) did not extend life span of genetically heterogeneous mice, while low-dose rapamycintreated mice $(2.24 \mathrm{mg} / \mathrm{kg}$ body weight/day) showed an increase in the life span.

4.2.2. The Effects of Resveratrol in Clinical Trials. Data of several clinical trials about resveratrol acting on cognition and learning and memory has indicated that resveratrol plays a protective role not only in diabetic patients but also in nondiabetic population except for patients with schizophrenia. In a randomized controlled trial on T2DM adults, a low dose of resveratrol ( $75 \mathrm{mg}$ at weekly intervals) showed a positive but chronic effect on cerebrovascular function and cognitive function $[123,124]$. In a 14-week randomized placebocontrolled intervention trial, resveratrol supplementation (75 mg twice daily) improved cognitive performance, mood, and cerebrovascular function in postmenopausal women [125]. However, resveratrol supplementation (200 mg/day for 1 month) did not improve memory and attention in 19 men with a diagnosis of schizophrenia [126].

Taken together, their findings have indicated that dosage and period of treatment may influence the effects of resveratrol. Compared to previous studies on antiaging and protection of cognitive decline, dosage of resveratrol and period of treatment are both insufficient. This idea suggests that resveratrol may not be the optimal choice for improvement of learning and memory in short-term treatment. So far, preclinical and clinical data in this area are limited, and an in-depth study of resveratrol on learning and memory needs to be further investigated.

\section{Conclusion}

SIRT1 may improve cognition and learning and memory through several pathways including deacetylation, OS, mitochondrial dysfunction, and inflammation and microRNA. However, the mechanisms of SIRT1 in cognitive decline under the condition of IR are inadequate and not in-depth and systematical, for example, a gap in the studies on mechanism of SIRT1 regulating neuronal energy metabolism and function. Meanwhile, the involvement of resveratrol, an activator of SIRT1, in the protection of cognitive deficits is still not completely clear. But in most cases, resveratrol can improve cognition and learning and memory. Thereby, the neuroprotection of SIRT1 and resveratrol and their interaction should be explored to act on preventing cognitive impairment in T2DM-associated cognitive dysfunction. In brief, SIRT1 may provide potential approaches to improve learning and memory. Long-term therapy of large-dose resveratrol may offer therapeutic possibilities for preventive strategies in T2DM-associated cognitive dysfunction.

\section{Conflicts of Interest}

The authors declare that there is no conflict of interest regarding the publication of this paper.

\section{Authors' Contributions}

Yue Cao and Guixia Wang designed this review, $\mathrm{Zi}$ Yan and Tong Zhou participated in revising the article and polishing the language, and Yue Cao and Guixia Wang wrote the manuscript and were responsible for the final content of the manuscript. All authors read and approved the final manuscript.

\section{Acknowledgments}

This work was supported by a grant (belonging to Guixia Wang) from the National Nature Science Foundation of China (Grant no. 81670732) and a grant (belonging to Yue Cao) from the Graduate Innovation Fund of Jilin University (Grant no. 2017095).

\section{References}

[1] C. Fritschi, U. G. Bronas, C. G. Park, E. G. Collins, and L. Quinn, "Early declines in physical function among aging 
adults with type 2 diabetes," Journal of Diabetes and its Complications, vol. 31, pp. 347-352, 2017.

[2] S. Yoon, H. Cho, J. Kim et al., "Brain changes in overweight/ obese and normal-weight adults with type 2 diabetes mellitus," Diabetologia, vol. 60, pp. 1207-1217, 2017.

[3] W. Li, T. Wang, and S. Xiao, "Type 2 diabetes mellitus might be a risk factor for mild cognitive impairment progressing to Alzheimer's disease," Neuropsychiatric Disease and Treatment, vol. 12, pp. 2489-2495, 2016.

[4] H. Ascher-Svanum, Y. F. Chen, A. Hake et al., "Cognitive and functional decline in patients with mild Alzheimer dementia with or without comorbid diabetes," Clinical Therapeutics, vol. 37, pp. 1195-1205, 2015.

[5] P. J. Fried, L. Schilberg, A. K. Brem et al., "Humans with type-2 diabetes show abnormal long-term potentiationlike cortical plasticity associated with verbal learning deficits," Journal of Alzheimer's Disease, vol. 55, pp. 89-100, 2017.

[6] K. Talbot, H. Y. Wang, H. Kazi et al., "Demonstrated brain insulin resistance in Alzheimer's disease patients is associated with IGF-1 resistance, IRS-1 dysregulation, and cognitive decline," The Journal of Clinical Investigation, vol. 122, pp. 1316-1338, 2012.

[7] M. W. Strachan, "R D Lawrence Lecture 2010. The brain as a target organ in type 2 diabetes: exploring the links with cognitive impairment and dementia," Diabetic Medicine, vol. 28, pp. 141-147, 2011.

[8] T. B. Chen, S. Y. Yiao, Y. Sun et al., "Comorbidity and dementia: a nationwide survey in Taiwan," PLoS One, vol. 12, article e0175475, 2017.

[9] B. J. North and E. Verdin, "Sirtuins: Sir2-related NADdependent protein deacetylases," Genome Biology, vol. 5, p. 224, 2004.

[10] M. Kitada, S. Kume, A. Takeda-Watanabe, K. Kanasaki, and D. Koya, "Sirtuins and renal diseases: relationship with aging and diabetic nephropathy," Clinical Science (London, England), vol. 124, pp. 153-164, 2013.

[11] M. Kaeberlein, M. McVey, and L. Guarente, “The SIR2/3/4 complex and SIR2 alone promote longevity in Saccharomyces cerevisiae by two different mechanisms," Genes \& Development, vol. 13, pp. 2570-2580, 1999.

[12] E. Michishita, J. Y. Park, J. M. Burneskis, J. C. Barrett, and I. Horikawa, "Evolutionarily conserved and nonconserved cellular localizations and functions of human SIRT proteins," Molecular Biology of the Cell, vol. 16, pp. 4623-4635, 2005.

[13] Y. Cao, X. Jiang, H. Ma, Y. Wang, P. Xue, and Y. Liu, "SIRT1 and insulin resistance," Journal of Diabetes and its Complications, vol. 30, pp. 178-183, 2016.

[14] T. Ogawa, C. Wakai, T. Saito et al., "Distribution of the longevity gene product, SIRT1, in developing mouse organs," Congenital Anomalies (Kyoto), vol. 51, pp. 70-79, 2011.

[15] S. M. Zakhary, D. Ayubcha, J. N. Dileo et al., "Distribution analysis of deacetylase SIRT1 in rodent and human nervous systems," Anatomical Record (Hoboken, N.J. : 2007), vol. 293, pp. 1024-1032, 2010.

[16] T. Shan, Y. Wang, T. Wu et al., "Porcine sirtuin 1 gene clone, expression pattern, and regulation by resveratrol," Journal of Animal Science, vol. 87, pp. 895-904, 2009.

[17] S. Michan, Y. Li, M. M. Chou et al., "SIRT1 is essential for normal cognitive function and synaptic plasticity," The Journal of Neuroscience, vol. 30, pp. 9695-9707, 2010.
[18] Q. Wang, C. Yan, M. Xin, L. Han, Y. Zhang, and M. Sun, "Sirtuin 1 (Sirt1) overexpression in BaF3 cells contributes to cell proliferation promotion, apoptosis resistance and pro-inflammatory cytokine production," Medical Science Monitor, vol. 23, pp. 1477-1482, 2017.

[19] A. Sathyanarayan, M. T. Mashek, and D. G. Mashek, "ATGL promotes autophagy/lipophagy via SIRT1 to control hepatic lipid droplet catabolism," Cell Reports, vol. 19, pp. 1-9, 2017.

[20] H. L. Cheng, R. Mostoslavsky, S. Saito et al., "Developmental defects and p53 hyperacetylation in Sir2 homolog (SIRT1)deficient mice," Proceedings of the National Academy of Sciences of the United States of America, vol. 100, pp. 10794-10799, 2003.

[21] K. Nagao, T. Jinnouchi, S. Kai, and T. Yanagita, "Pterostilbene, a dimethylated analog of resveratrol, promotes energy metabolism in obese rats," The Journal of Nutritional Biochemistry, vol. 43, pp. 151-155, 2017.

[22] H. J. Lee and S. J. Yang, “Aging-related correlation between serum sirtuin 1 activities and basal metabolic rate in women, but not in men," Clinical Nutrition Research, vol. 6, pp. 1826, 2017.

[23] M. M. Bellet, S. Masri, G. Astarita, P. Sassone-Corsi, M. A. Della Fazia, and G. Servillo, "Histone deacetylase SIRT1 controls proliferation, circadian rhythm, and lipid metabolism during liver regeneration in mice," The Journal of Biological Chemistry, vol. 291, pp. 23318-23329, 2016.

[24] R. H. Wang, T. Zhao, K. Cui et al., "Negative reciprocal regulation between Sirt1 and Per2 modulates the circadian clock and aging," Scientific Reports, vol. 6, article 28633, 2016.

[25] M. C. Haigis and D. A. Sinclair, "Mammalian sirtuins: biological insights and disease relevance," Annual Review of Pathology, vol. 5, pp. 253-295, 2010.

[26] S. Fusco, G. Maulucci, and G. Pani, "Sirt1: def-eating senescence?," Cell Cycle, vol. 11, pp. 4135-4146, 2012.

[27] W. Grabowska, E. Sikora, and A. Bielak-Zmijewska, "Sirtuins, a promising target in slowing down the ageing process," Biogerontology, vol. 18, no. 4, pp. 447-476, 2017.

[28] D. F. Silva, J. E. Selfridge, J. Lu et al., "Bioenergetic flux, mitochondrial mass and mitochondrial morphology dynamics in AD and MCI cybrid cell lines," Human Molecular Genetics, vol. 22, pp. 3931-3946, 2013.

[29] S. H. Cho, J. A. Chen, F. Sayed et al., "SIRT1 deficiency in microglia contributes to cognitive decline in aging and neurodegeneration via epigenetic regulation of IL-1beta," The Journal of Neuroscience, vol. 35, pp. 807-818, 2015.

[30] Y. Y. Chen, L. Zhang, D. L. Shi et al., "Resveratrol attenuates subacute systemic inflammation-induced spatial memory impairment via inhibition of astrocyte activation and enhancement of synaptophysin expression in the hippocampus," Annals of Clinical and Laboratory Science, vol. 47, pp. 17-24, 2017.

[31] R. Wang, Y. Zhang, J. Li, and C. Zhang, "Resveratrol ameliorates spatial learning memory impairment induced by Abeta1-42 in rats," Neuroscience, vol. 344, pp. 39-47, 2017.

[32] Y. Nomura and Y. Okuma, "Age-related defects in lifespan and learning ability in SAMP8 mice," Neurobiology of Aging, vol. 20, pp. 111-115, 1999.

[33] T. Takeda, "Senescence-accelerated mouse (SAM) with special references to neurodegeneration models, SAMP8 
and SAMP10 mice," Neurochemical Research, vol. 34, pp. 639-659, 2009.

[34] J. C. Lopez-Ramos, M. T. Jurado-Parras, C. Sanfeliu, D. Acuña-Castroviejo, and J. M. Delgado-García, "Learning capabilities and CA1-prefrontal synaptic plasticity in a mice model of accelerated senescence," Neurobiology of Aging, vol. 33, article 627.e613-626, 2012.

[35] X. R. Cheng, X. L. Cui, Y. Zheng et al., "Nodes and biological processes identified on the basis of network analysis in the brain of the senescence accelerated mice as an Alzheimer's disease animal model," Frontiers in Aging Neuroscience, vol. 5, p. 65, 2013.

[36] X. R. Cheng, W. X. Zhou, and Y. X. Zhang, "The behavioral, pathological and therapeutic features of the senescenceaccelerated mouse prone 8 strain as an Alzheimer's disease animal model," Ageing Research Reviews, vol. 13, pp. 1337, 2014

[37] J. E. Morley, H. J. Armbrecht, S. A. Farr, and V. B. Kumar, "The senescence accelerated mouse (SAMP8) as a model for oxidative stress and Alzheimer's disease," Biochimica et Biophysica Acta, vol. 1822, pp. 650-656, 2012.

[38] J. E. Morley, S. A. Farr, V. B. Kumar, and H. J. Armbrecht, "The SAMP8 mouse: a model to develop therapeutic interventions for Alzheimer's disease," Current Pharmaceutical Design, vol. 18, pp. 1123-1130, 2012.

[39] H. Wang, K. Lian, B. Han et al., "Age-related alterations in the metabolic profile in the hippocampus of the senescenceaccelerated mouse prone 8: a spontaneous Alzheimer's disease mouse model," Journal of Alzheimer's Disease, vol. 39, pp. 841-848, 2014

[40] H. Gong, J. Pang, Y. Han et al., "Age-dependent tissue expression patterns of Sirt1 in senescence-accelerated mice," Molecular Medicine Reports, vol. 10, pp. 3296-3302, 2014.

[41] T. Takeda, M. Hosokawa, and K. Higuchi, "Senescenceaccelerated mouse (SAM): a novel murine model of accelerated senescence," Journal of the American Geriatrics Society, vol. 39, pp. 911-919, 1991.

[42] M. Cosin-Tomas, M. J. Alvarez-Lopez, S. Sanchez-Roige et al., "Epigenetic alterations in hippocampus of SAMP8 senescent mice and modulation by voluntary physical exercise," Frontiers in Aging Neuroscience, vol. 6, p. 51, 2014.

[43] H. S. Kim, S. Moon, J. H. Paik et al., "Activation of the $5^{\prime}$-AMP-activated protein kinase in the cerebral cortex of young senescence-accelerated P8 mice and association with GSK3 $\beta$ - and PP2A-dependent inhibition of $\mathrm{p}$ - $\mathrm{tau}_{396}$ expression," Journal of Alzheimer's Disease, vol. 46, pp. 249259, 2015.

[44] M. Pallas, J. G. Pizarro, J. Gutierrez-Cuesta et al., "Modulation of SIRT1 expression in different neurodegenerative models and human pathologies," Neuroscience, vol. 154, pp. 1388-1397, 2008.

[45] B. L. Xu, R. Wang, L. N. Ma et al., "Effects of caloric intake on learning and memory function in juvenile C57BL/6J mice," BioMed Research International, vol. 2015, Article ID 759803, 7 pages, 2015.

[46] Y. A. Lee, E. J. Cho, and T. Yokozawa, "Protective effect of persimmon (Diospyros kaki) peel proanthocyanidin against oxidative damage under $\mathrm{H} 2 \mathrm{O} 2$-induced cellular senescence," Biological \& Pharmaceutical Bulletin, vol. 31, pp. 12651269, 2008.
[47] T. Yokozawa, Y. A. Lee, E. J. Cho, K. Matsumoto, C. H. Park, and N. Shibahara, "Anti-aging effects of oligomeric proanthocyanidins isolated from persimmon fruits," Drug Discoveries \& Therapeutics, vol. 5, pp. 109-118, 2011.

[48] K. Kakefuda, Y. Fujita, A. Oyagi et al., "Sirtuin 1 overexpression mice show a reference memory deficit, but not neuroprotection," Biochemical and Biophysical Research Communications, vol. 387, pp. 784-788, 2009.

[49] K. N. Green, J. S. Steffan, H. Martinez-Coria et al., "Nicotinamide restores cognition in Alzheimer's disease transgenic mice via a mechanism involving sirtuin inhibition and selective reduction of Thr231-phosphotau," The Journal of Neuroscience, vol. 28, pp. 11500-11510, 2008.

[50] L. Sansone, V. Reali, L. Pellegrini et al., "SIRT1 silencing confers neuroprotection through IGF-1 pathway activation," Journal of Cellular Physiology, vol. 228, pp. 1754-1761, 2013.

[51] A. Vaquero, M. Scher, D. Lee, H. Erdjument-Bromage, P. Tempst, and D. Reinberg, "Human SirT1 interacts with histone $\mathrm{H} 1$ and promotes formation of facultative heterochromatin," Molecular Cell, vol. 16, pp. 93-105, 2004.

[52] H. Vaziri, S. K. Dessain, E. Ng Eaton et al., "hSIR2 ${ }^{\text {SIRT1 }}$ functions as an NAD-dependent p53 deacetylase," Cell, vol. 107, pp. 149-159, 2001.

[53] J. Luo, A. Y. Nikolaev, S. Imai et al., "Negative control of p53 by Sir2alpha promotes cell survival under stress," Cell, vol. 107, pp. 137-148, 2001.

[54] S. Bayod, C. Guzman-Brambila, S. Sanchez-Roige et al., "Voluntary exercise promotes beneficial anti-aging mechanisms in SAMP8 female brain," Journal of Molecular Neuroscience, vol. 55, pp. 525-532, 2015.

[55] D. Porquet, G. Casadesus, S. Bayod et al., "Dietary resveratrol prevents Alzheimer's markers and increases life span in SAMP8," Age (Dordrecht, Netherlands), vol. 35, pp. 18511865, 2013.

[56] R. Cristofol, D. Porquet, R. Corpas et al., "Neurons from senescence-accelerated SAMP8 mice are protected against frailty by the sirtuin 1 promoting agents melatonin and resveratrol," Journal of Pineal Research, vol. 52, pp. 271281, 2012.

[57] B. L. Xu, R. Wang, L. N. Ma et al., "Comparison of the effects of resveratrol and caloric restriction on learning and memory in juvenile C57BL/6J mice," Iranian Journal of Basic Medical Sciences, vol. 18, pp. 1118-1123, 2015.

[58] Z. Zheng, H. Chen, J. Li et al., "Sirtuin 1-mediated cellular metabolic memory of high glucose via the LKB1/AMPK/ ROS pathway and therapeutic effects of metformin," Diabetes, vol. 61, pp. 217-228, 2012.

[59] C. Canto, Z. Gerhart-Hines, J. N. Feige et al., "AMPK regulates energy expenditure by modulating $\mathrm{NAD}^{+}$metabolism and SIRT1 activity," Nature, vol. 458, pp. 1056-1060, 2009.

[60] L. L. Du, D. M. Chai, L. N. Zhao et al., "AMPK activation ameliorates Alzheimer's disease-like pathology and spatial memory impairment in a streptozotocin-induced Alzheimer's disease model in rats," Journal of Alzheimer's Disease, vol. 43, pp. 775-784, 2015.

[61] K. J. Pearson, J. A. Baur, K. N. Lewis et al., "Resveratrol delays age-related deterioration and mimics transcriptional aspects of dietary restriction without extending life span," Cell Metabolism, vol. 8, pp. 157-168, 2008.

[62] M. Khan, S. A. Shah, and M. O. Kim, "17 $\beta$-estradiol via SIRT1/ acetyl-p53/NF-kB signaling pathway rescued postnatal rat 
brain against acute ethanol intoxication," Molecular Neurobiology, pp. 1-12, 2017.

[63] F. Lan, J. M. Cacicedo, N. Ruderman, and Y. Ido, "SIRT1 modulation of the acetylation status, cytosolic localization, and activity of LKB1. Possible role in AMP-activated protein kinase activation," The Journal of Biological Chemistry, vol. 283, pp. 27628-27635, 2008.

[64] A. Salminen, J. Huuskonen, J. Ojala, A. Kauppinen, K. Kaarniranta, and T. Suuronen, "Activation of innate immunity system during aging: NF-kB signaling is the molecular culprit of inflamm-aging," Ageing Research Reviews, vol. 7, pp. 83-105, 2008.

[65] L. L. Du, J. Z. Xie, X. S. Cheng et al., "Activation of sirtuin 1 attenuates cerebral ventricular streptozotocin-induced tau hyperphosphorylation and cognitive injuries in rat hippocampi," Age (Dordrecht, Netherlands), vol. 36, pp. 613623, 2014.

[66] Y. I. Kitamura, T. Kitamura, J. P. Kruse et al., "FoxO1 protects against pancreatic beta cell failure through NeuroD and MafA induction," Cell Metabolism, vol. 2, pp. 153-163, 2005.

[67] H. Y. Cohen, C. Miller, K. J. Bitterman et al., "Calorie restriction promotes mammalian cell survival by inducing the SIRT1 deacetylase," Science, vol. 305, pp. 390-392, 2004.

[68] J. T. Rodgers, C. Lerin, W. Haas, S. P. Gygi, B. M. Spiegelman, and P. Puigserver, "Nutrient control of glucose homeostasis through a complex of PGC-1alpha and SIRT1," Nature, vol. 434, pp. 113-118, 2005.

[69] L. R. Saunders, A. D. Sharma, J. Tawney et al., "miRNAs regulate SIRT1 expression during mouse embryonic stem cell differentiation and in adult mouse tissues," Aging (Albany NY), vol. 2, pp. 415-431, 2010.

[70] M. Tajes, J. Gutierrez-Cuesta, J. Folch et al., "Neuroprotective role of intermittent fasting in senescence-accelerated mice P8 (SAMP8)," Experimental Gerontology, vol. 45, pp. 702710, 2010.

[71] J. Gutierrez-Cuesta, M. Tajes, A. Jimenez, A. Coto-Montes, A. Camins, and M. Pallàs, "Evaluation of potential prosurvival pathways regulated by melatonin in a murine senescence model," Journal of Pineal Research, vol. 45, pp. 497-505, 2008.

[72] R. Corpas, S. Revilla, S. Ursulet et al., "SIRT1 overexpression in mouse hippocampus induces cognitive enhancement through proteostatic and neurotrophic mechanisms," Molecular Neurobiology, vol. 54, no. 7, pp. 5604-5619, 2017.

[73] H. R. Lee, H. K. Shin, S. Y. Park et al., "Cilostazol suppresses $\beta$-amyloid production by activating a disintegrin and metalloproteinase 10 via the upregulation of SIRT1-coupled retinoic acid receptor- $\beta$," Journal of Neuroscience Research, vol. 92, pp. 1581-1590, 2014.

[74] V. L. Villemagne, V. Dore, P. Bourgeat et al., "Abeta-amyloid and tau imaging in dementia," Seminars in Nuclear Medicine, vol. 47, pp. 75-88, 2017.

[75] W. Guo, L. Qian, J. Zhang et al., "Sirt1 overexpression in neurons promotes neurite outgrowth and cell survival through inhibition of the mTOR signaling," Journal of Neuroscience Research, vol. 89, pp. 1723-1736, 2011.

[76] J. F. Codocedo, C. Allard, J. A. Godoy, L. Varela-Nallar, and N. C. Inestrosa, "SIRT1 regulates dendritic development in hippocampal neurons," PLoS One, vol. 7, article e47073, 2012.

[77] M. F. Xu, Y. Y. Xiong, J. K. Liu, J. J. Qian, L. Zhu, and J. Gao, "Asiatic acid, a pentacyclic triterpene in Centella asiatica, attenuates glutamate-induced cognitive deficits in mice and apoptosis in SH-SY5Y cells," Acta Pharmacologica Sinica, vol. 33, pp. 578-587, 2012.

[78] I. B. Zovkic, B. S. Paulukaitis, J. J. Day, D. M. Etikala, and J. D. Sweatt, "Histone H2A.Z subunit exchange controls consolidation of recent and remote memory," Nature, vol. 515, pp. 582-586, 2014.

[79] T. Baptista, I. Graca, E. J. Sousa et al., "Regulation of histone H2A.Z expression is mediated by sirtuin 1 in prostate cancer," Oncotarget, vol. 4, pp. 1673-1685, 2013.

[80] K. S. Petersen and C. Smith, "Ageing-associated oxidative stress and inflammation are alleviated by products from grapes," Oxidative Medicine and Cellular Longevity, vol. 2016, Article ID 6236309, 12 pages, 2016.

[81] S. Garcia-Matas, N. Verade, A. O. Aznar et al., "In vitro and in vivo activation of astrocytes by amyloid- $\beta$ is potentiated by pro-oxidant agents," Journal of Alzheimer's Disease, vol. 20, pp. 229-245, 2010.

[82] R. Quiroz-Baez, D. Flores-Dominguez, and C. Arias, "Synaptic aging is associated with mitochondrial dysfunction, reduced antioxidant contents and increased vulnerability to amyloid-beta toxicity," Current Alzheimer Research, vol. 10, pp. 324-331, 2013.

[83] A. Navarro and A. Boveris, "The mitochondrial energy transduction system and the aging process," American Journal of Physiology. Cell Physiology, vol. 292, pp. C670C686, 2007.

[84] J. Valledel, J. Duran-Vilaregut, G. Manich et al., "Cerebral amyloid angiopathy, blood-brain barrier disruption and amyloid accumulation in SAMP8 mice," Neurodegenerative Diseases, vol. 8, pp. 421-429, 2011.

[85] H. Ota, M. Akishita, T. Akiyoshi et al., "Testosterone deficiency accelerates neuronal and vascular aging of SAMP8 mice: protective role of eNOS and SIRT1," PLoS One, vol. 7, article e29598, 2012.

[86] T. Wang, G. Di, L. Yang et al., "Saponins from Panax japonicus attenuate D-galactose-induced cognitive impairment through its anti-oxidative and anti-apoptotic effects in rats," The Journal of Pharmacy and Pharmacology, vol. 67, pp. 1284-1296, 2015.

[87] J. Liu, G. Hu, R. Xu et al., "Rhein lysinate decreases the generation of $\beta$-amyloid in the brain tissues of Alzheimer's disease model mice by inhibiting inflammatory response and oxidative stress," Journal of Asian Natural Products Research, vol. 15, pp. 756-763, 2013.

[88] H. M. Wang, L. W. Wang, X. M. Liu, C. L. Li, S. P. Xu, and A. D. Farooq, "Neuroprotective effects of forsythiaside on learning and memory deficits in senescence-accelerated mouse prone (SAMP8) mice," Pharmacology, Biochemistry, and Behavior, vol. 105, pp. 134-141, 2013.

[89] Y. Yoshiyama, M. Higuchi, B. Zhang et al., "Synapse loss and microglial activation precede tangles in a P301S tauopathy mouse model," Neuron, vol. 53, pp. 337-351, 2007.

[90] A. Z. Herskovits and L. Guarente, "SIRT1 in neurodevelopment and brain senescence," Neuron, vol. 81, pp. 471483, 2014.

[91] J. Gao, W. Y. Wang, Y. W. Mao et al., "A novel pathway regulates memory and plasticity via SIRT1 and miR-134," Nature, vol. 466, pp. 1105-1109, 2010.

[92] Y. N. Zhao, W. F. Li, F. Li et al., "Resveratrol improves learning and memory in normally aged mice through 
microRNA-CREB pathway," Biochemical and Biophysical Research Communications, vol. 435, pp. 597-602, 2013.

[93] C. Feng, J. Gu, F. Zhou et al., "The effect of lead exposure on expression of SIRT1 in the rat hippocampus," Environmental Toxicology and Pharmacology, vol. 44, pp. 84-92, 2016.

[94] A. Cardoso, F. Marrana, and J. P. Andrade, "Caloric restriction in young rats disturbs hippocampal neurogenesis and spatial learning," Neurobiology of Learning and Memory, vol. 133, pp. 214-224, 2016.

[95] P. S. Koekkoek, L. J. Kappelle, E. van den Berg, G. E. Rutten, and G. J. Biessels, "Cognitive function in patients with diabetes mellitus: guidance for daily care," Lancet Neurology, vol. 14, pp. 329-340, 2015.

[96] D. Qi, A. Wang, Y. Chen et al., "Default mode network connectivity and related white matter disruption in type 2 diabetes mellitus patients concurrent with amnestic mild cognitive impairment," Current Alzheimer Research, 2017.

[97] J. F. Jansen, F. C. Busselvan, H. J. Haarvan de et al., "Cerebral blood flow, blood supply, and cognition in type 2 diabetes mellitus," Scientific Reports, vol. 6, p. 10, 2016.

[98] R. O. Roberts, D. S. Knopman, Y. E. Geda et al., "Association of diabetes with amnestic and nonamnestic mild cognitive impairment," Alzheimers Dement, vol. 10, pp. 18-26, 2014.

[99] C. C. Chung, D. Pimentel, A. J. Jor'dan, Y. Hao, W. Milberg, and V. Novak, "Inflammation-associated declines in cerebral vasoreactivity and cognition in type 2 diabetes," Neurology, vol. 85, pp. 450-458, 2015.

[100] J. Mehla, B. C. Chauhan, and N. B. Chauhan, "Experimental induction of type 2 diabetes in aging-accelerated mice triggered Alzheimer-like pathology and memory deficits," Journal of Alzheimer's Disease, vol. 39, pp. 145-162, 2014.

[101] D. Sweetnam, A. Holmes, K. A. Tennant et al., "Diabetes impairs cortical plasticity and functional recovery following ischemic stroke," The Journal of Neuroscience, vol. 32, pp. 5132-5143, 2012.

[102] V. Palomera-Avalos, C. Grinan-Ferre, V. Izquierdo, A. Camins, C. Sanfeliu, and M. Pallàs, "Metabolic stress induces cognitive disturbances and inflammation in aged mice: protective role of resveratrol," Rejuvenation Research, vol. 20, no. 3, pp. 202-217, 2017.

[103] V. Palomera-Avalos, C. Grinan-Ferre, D. Puigoriol-Ilamola et al., "Resveratrol protects SAMP8 brain under metabolic stress: focus on mitochondrial function and Wnt pathway," Molecular Neurobiology, vol. 54, no. 3, pp. 1661-1676, 2017.

[104] R. H. Wang, H. S. Kim, C. Xiao, X. Xu, O. Gavrilova, and C. X. Deng, "Hepatic Sirt1 deficiency in mice impairs mTorc2/Akt signaling and results in hyperglycemia, oxidative damage, and insulin resistance," The Journal of Clinical Investigation, vol. 121, pp. 4477-4490, 2011.

[105] B. Zhou, C. Li, W. Qi et al., "Downregulation of miR-181a upregulates sirtuin-1 (SIRT1) and improves hepatic insulin sensitivity," Diabetologia, vol. 55, pp. 2032-2043, 2012.

[106] S. Frojdo, C. Durand, L. Molin et al., "Phosphoinositide 3kinase as a novel functional target for the regulation of the insulin signaling pathway by SIRT1," Molecular and Cellular Endocrinology, vol. 335, pp. 166-176, 2011.

[107] A. Chalkiadaki and L. Guarente, "High-fat diet triggers inflammation-induced cleavage of SIRT1 in adipose tissue to promote metabolic dysfunction," Cell Metabolism, vol. 16, pp. 180-188, 2012.
[108] B. Draznin, "Molecular mechanisms of insulin resistance: serine phosphorylation of insulin receptor substrate- 1 and increased expression of $\mathrm{p} 85 \alpha$ : the two sides of a coin," Diabetes, vol. 55, pp. 2392-2397, 2006.

[109] Y. Liu, Z. Yao, L. Zhang, H. Zhu, W. Deng, and C. Qin, "Insulin induces neurite outgrowth via SIRT1 in SH-SY5Y cells," Neuroscience, vol. 238, pp. 371-380, 2013.

[110] M. Uittenbogaard, K. K. Baxter, and A. Chiaramello, "The neurogenic basic helix-loop-helix transcription factor NeuroD6 confers tolerance to oxidative stress by triggering an antioxidant response and sustaining the mitochondrial biomass," ASN Neuro, vol. 2, article e00034, 2010.

[111] R. Lennox, D. W. Porter, P. R. Flatt, C. Holscher, N. Irwin, and V. A. Gault, "Comparison of the independent and combined effects of sub-chronic therapy with metformin and a stable GLP-1 receptor agonist on cognitive function, hippocampal synaptic plasticity and metabolic control in high-fat fed mice," Neuropharmacology, vol. 86, pp. 2230, 2014.

[112] N. M. Pathak, V. Pathak, A. M. Lynch, N. Irwin, V. A. Gault, and P. R. Flatt, "Stable oxyntomodulin analogues exert positive effects on hippocampal neurogenesis and gene expression as well as improving glucose homeostasis in high fat fed mice," Molecular and Cellular Endocrinology, vol. 412, pp. 95-103, 2015.

[113] M. Mendez-del Villar, M. Gonzalez-Ortiz, E. MartinezAbundis, K. G. Pérez-Rubio, and R. Lizárraga-Valdez, "Effect of resveratrol administration on metabolic syndrome, insulin sensitivity, and insulin secretion," Metabolic Syndrome and Related Disorders, vol. 12, pp. 497-501, 2014.

[114] J. Wang, C. Tang, M. G. Ferruzzi et al., "Role of standardized grape polyphenol preparation as a novel treatment to improve synaptic plasticity through attenuation of features of metabolic syndrome in a mouse model," Molecular Nutrition \& Food Research, vol. 57, pp. 2091-2102, 2013.

[115] S. Bastianetto, C. Menard, and R. Quirion, "Neuroprotective action of resveratrol," Biochimica et Biophysica Acta, vol. 1852, pp. 1195-1201, 2015.

[116] M. Kodali, V. K. Parihar, B. Hattiangady, V. Mishra, B. Shuai, and A. K. Shetty, "Resveratrol prevents age-related memory and mood dysfunction with increased hippocampal neurogenesis and microvasculature, and reduced glial activation," Scientific Reports, vol. 5, p. 8075, 2015.

[117] D. Bonnefont-Rousselot, "Resveratrol and cardiovascular diseases," Nutrients, vol. 8, 2016.

[118] P. C. Tang, Y. F. Ng, S. Ho, M. Gyda, and S. W. Chan, "Resveratrol and cardiovascular health - promising therapeutic or hopeless illusion?," Pharmacological Research, vol. 90, pp. 88-115, 2014.

[119] G. Wang, S. Amato, J. Gilbert, and H. Y. Man, "Resveratrol up-regulates AMPA receptor expression via AMP-activated protein kinase-mediated protein translation," Neuropharmacology, vol. 95, pp. 144-153, 2015.

[120] X. M. Li, M. T. Zhou, X. M. Wang, M. H. Ji, Z. Q. Zhou, and J. J. Yang, "Resveratrol pretreatment attenuates the isoflurane-induced cognitive impairment through its antiinflammation and -apoptosis actions in aged mice," Journal of Molecular Neuroscience, vol. 52, pp. 286-293, 2014.

[121] J. Chang, A. Rimando, M. Pallas et al., "Low-dose pterostilbene, but not resveratrol, is a potent neuromodulator in aging and Alzheimer's disease," Neurobiology of Aging, vol. 33, pp. 2062-2071, 2012. 
[122] R. A. Miller, D. E. Harrison, C. M. Astle et al., "Rapamycin, but not resveratrol or simvastatin, extends life span of genetically heterogeneous mice," The Journals of Gerontology. Series A, Biological Sciences and Medical Sciences, vol. 66, pp. 191-201, 2011.

[123] R. H. Wong, D. Raederstorff, and P. R. Howe, “Acute resveratrol consumption improves neurovascular coupling capacity in adults with type 2 diabetes mellitus," Nutrients, vol. 8, 2016.

[124] R. H. Wong, R. S. Nealon, A. Scholey, and P. R. Howe, "Low dose resveratrol improves cerebrovascular function in type 2 diabetes mellitus," Nutrition, Metabolism, and Cardiovascular Diseases, vol. 26, pp. 393-399, 2016.

[125] H. M. Evans, P. R. Howe, and R. H. Wong, "Effects of resveratrol on cognitive performance, mood and cerebrovascular function in post-menopausal women; a 14-week randomised placebo-controlled intervention trial," Nutrients, vol. 9, 2017.

[126] K. Zortea, V. C. Franco, P. Guimaraes, and P. S. Belmonte-deAbreu, "Resveratrol supplementation did not improve cognition in patients with schizophrenia: results from a randomized clinical trial," Frontiers in Psychiatry, vol. 7, p. 159, 2016. 


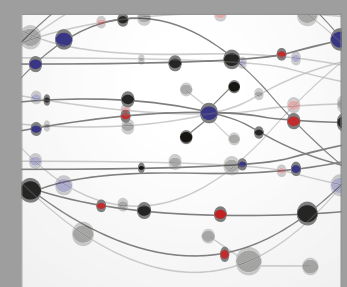

The Scientific World Journal
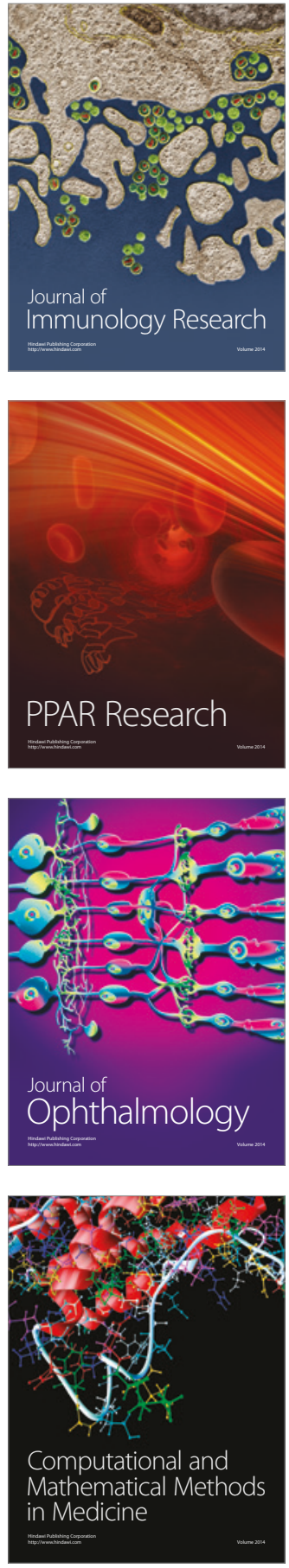

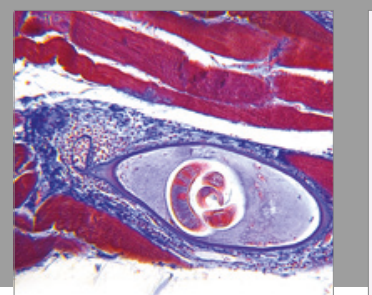

Gastroenterology Research and Practice
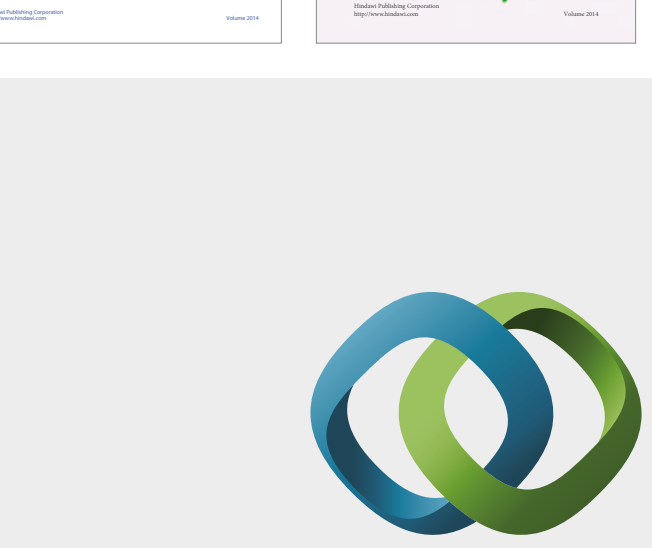

\section{Hindawi}

Submit your manuscripts at

https://www.hindawi.com
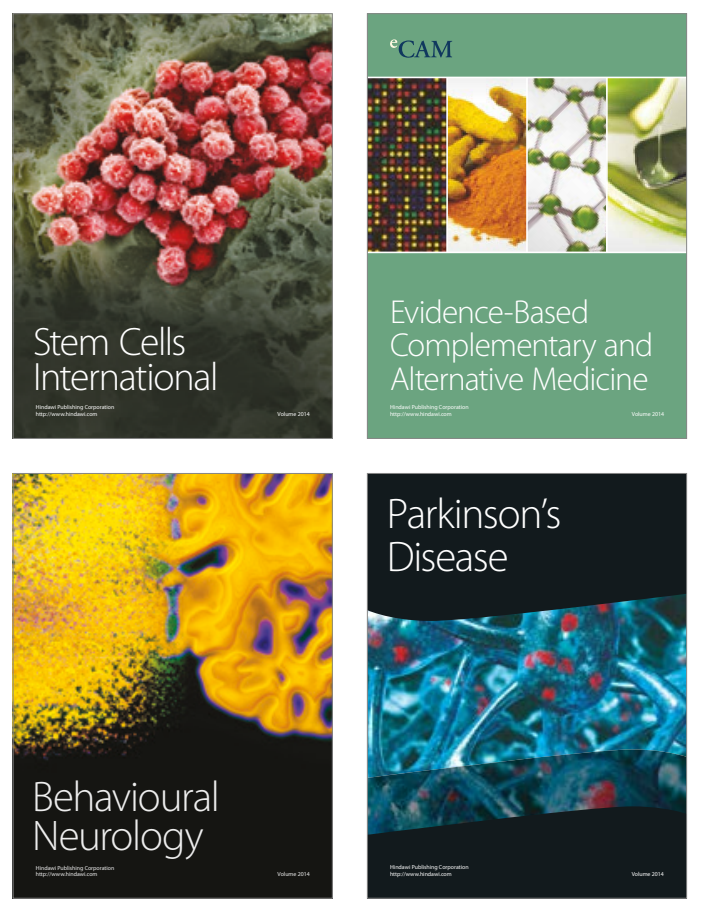
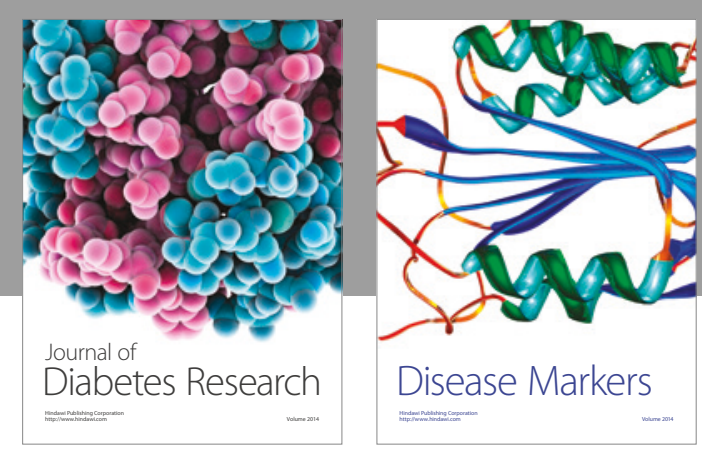

Disease Markers
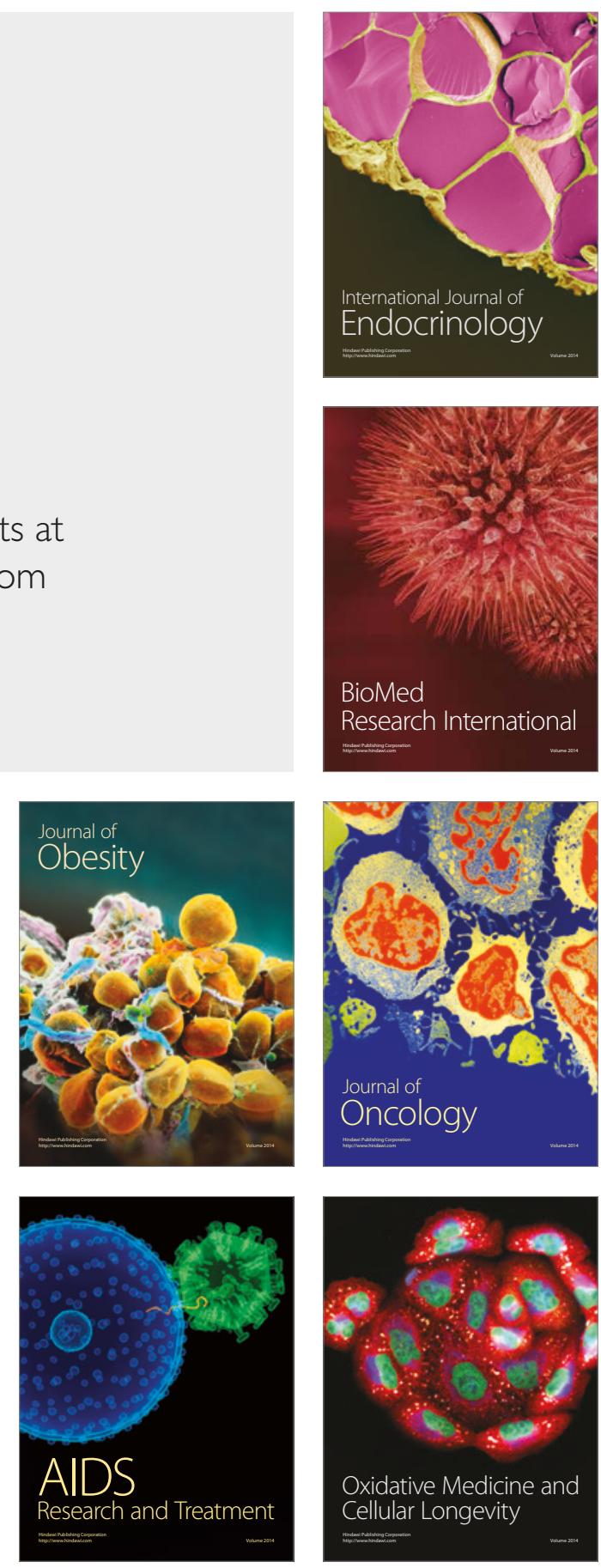